\title{
-Supporting Information
}

\section{Uniform Nano-SnO2/C Composite Anodes from Coal Tar Pitch for Sodium-Ion Batteries}

Yaoyu Li, Ruifeng Liu, Chunlei Wang, Ying Zhou*

College of Chemical Engineering, Dalian University of Technology, Dalian, Liaoning, 116023, P. R. China

* E-mail: zhouying.dlut@dlut.edu.cn (Y.Zhou)
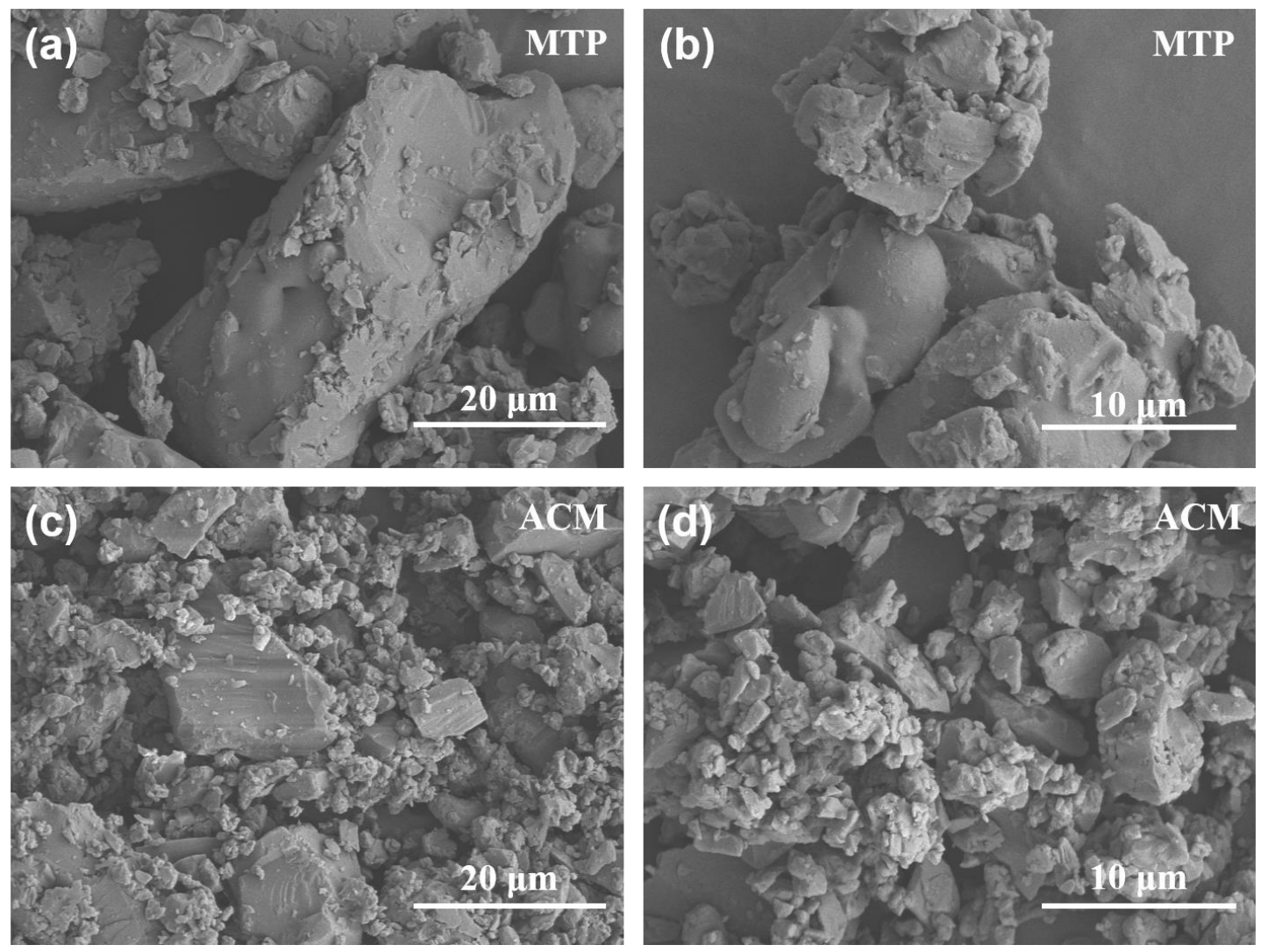

Figure S1. The SEM images of $(a, b)$ MTP and (c,d) ACM. 

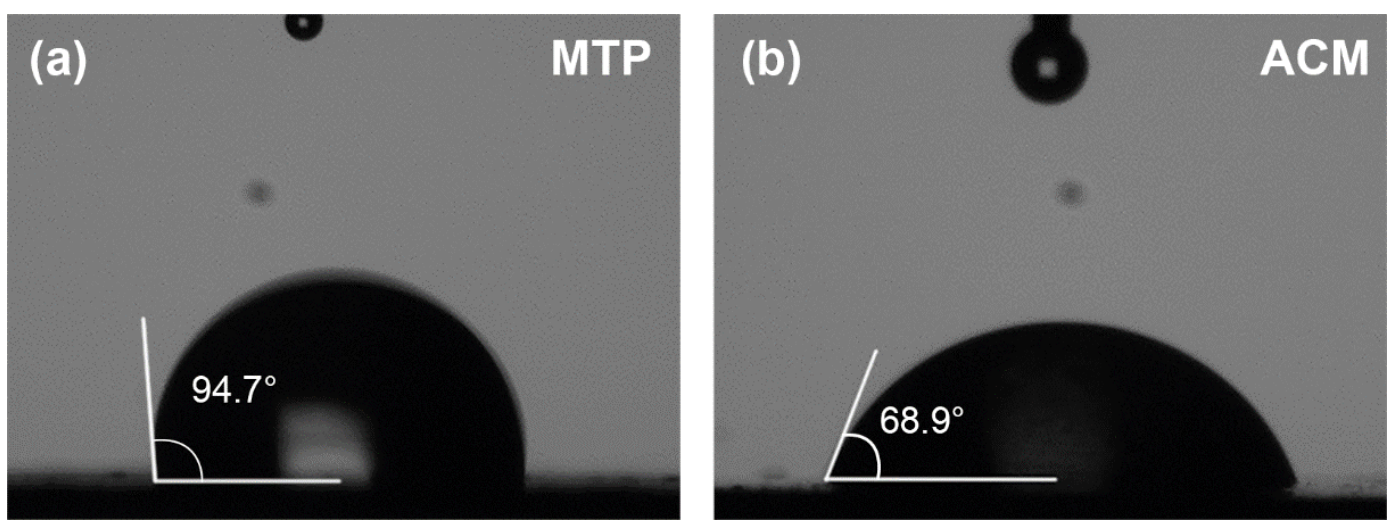

Figure S2. The water contact angle of (a) MTP and (b) ACM.
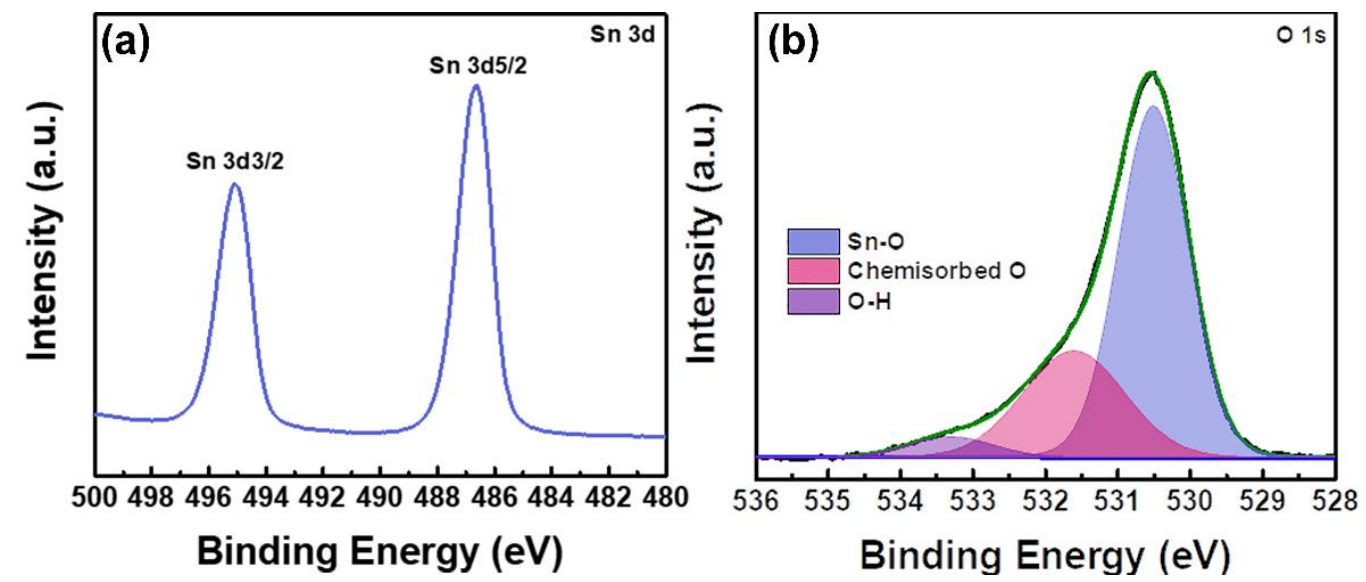

Figure S3. High resolution XPS spectra of $\mathrm{SnO}_{2}$ powder.
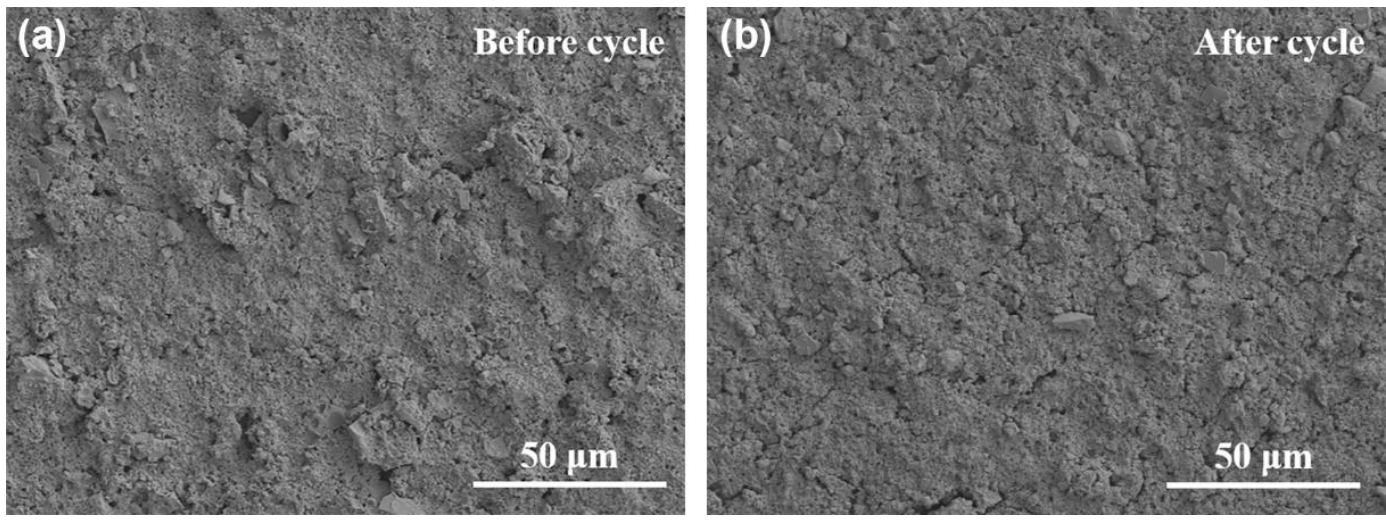

Figure S4. The SEM images of $\mathrm{SnO} 2 / \mathrm{C}-2$ electrode (a) before and (b) after 100 cycles. 


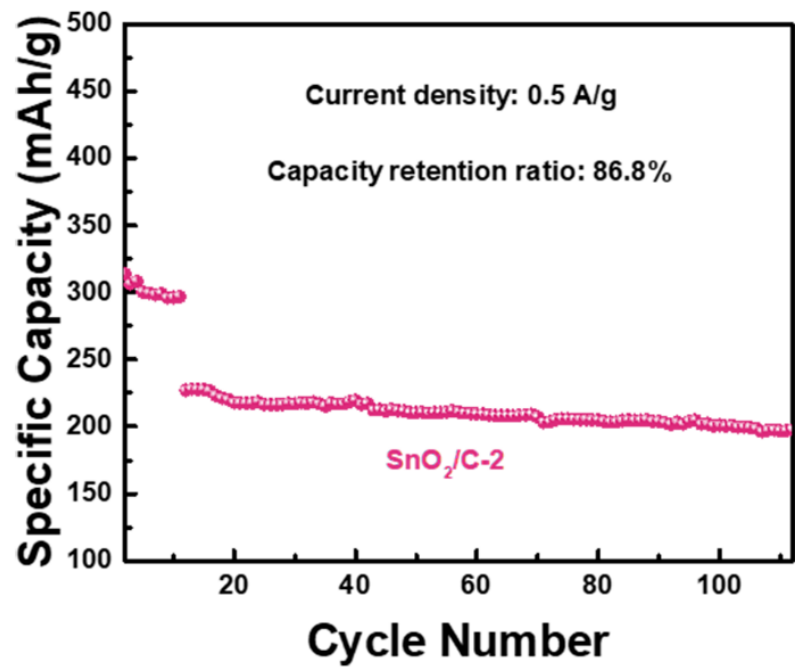

Figure S5. The cycling performance of $\mathrm{SnO} 2 / \mathrm{C}-2$ at $0.5 \mathrm{~A} / \mathrm{g}$ for 100 cycles.
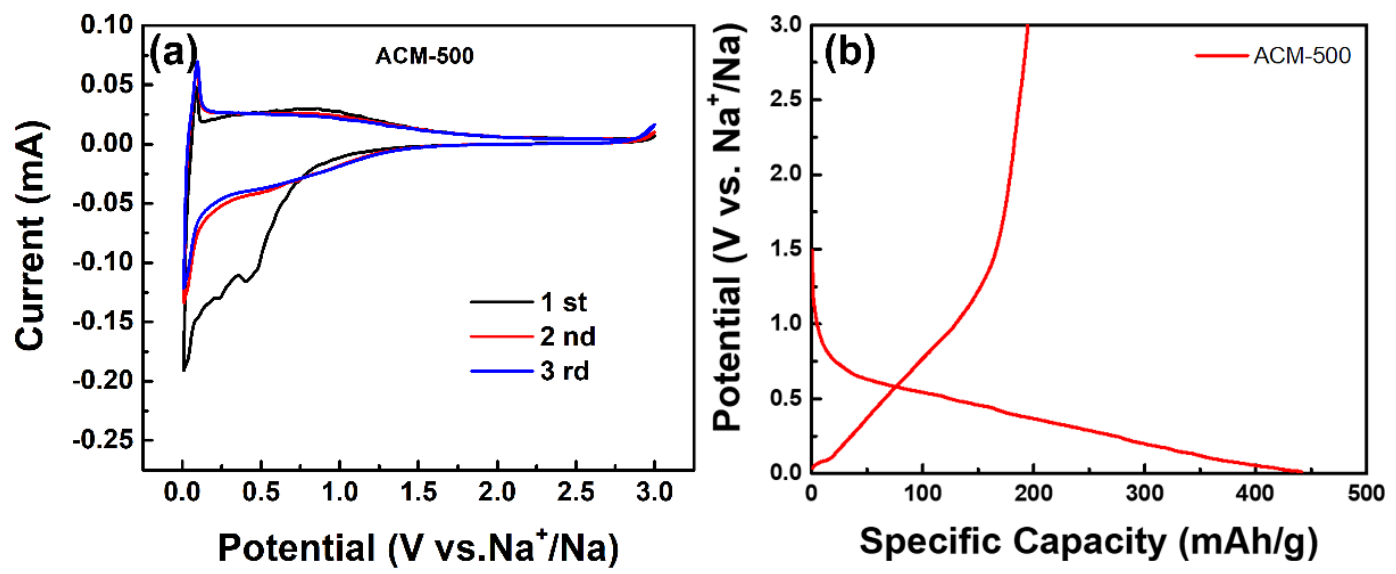

Figure S6. (a) the CV curves and (b) the charge/discharge curves of ACM-500.

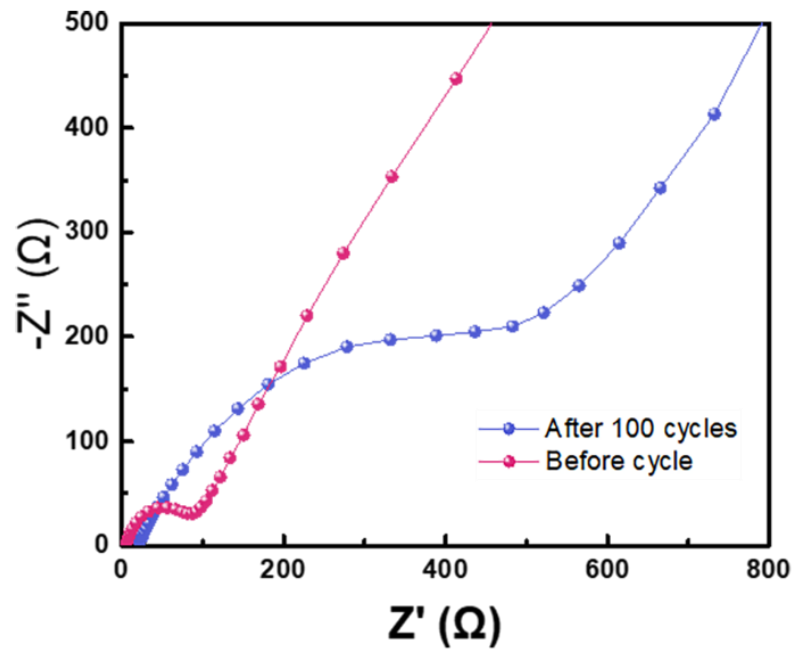


Figure S7. The EIS spectra of SnO2/C-2 anode before and after cycle. 